Itinéraires Itinéraires

Littérature, textes, cultures

2010-3 | 2010

Médiévalisme

\title{
Le passé sans l'histoire. Vers une anthropologie culturelle du temps
}

\section{Gil Bartholeyns}

\section{(2) OpenEdition}

\section{Journals}

Édition électronique

URL : http://journals.openedition.org/itineraires/1808

DOI : 10.4000/itineraires.1808

ISSN : 2427-920X

Éditeur

Pléiade

\section{Édition imprimée}

Date de publication : 1 novembre 2010

Pagination : 47-60

ISBN : 978-2-296-13150-7

ISSN : 2100-1340

\section{Référence électronique}

Gil Bartholeyns, «Le passé sans I'histoire. Vers une anthropologie culturelle du temps », Itinéraires [En ligne], 2010-3 | 2010, mis en ligne le 01 novembre 2010, consulté le 01 mai 2019. URL : http:// journals.openedition.org/itineraires/1808; DOI : 10.4000/itineraires.1808

\section{(ब) $(\Theta \Theta$}

Itinéraires est mis à disposition selon les termes de la licence Creative Commons Attribution - Pas d'Utilisation Commerciale - Pas de Modification 4.0 International. 


\title{
Le passé sans l'histoire. Vers une anthropologie culturelle du temps
}

\begin{abstract}
Historians live in the belief that history and memory are the only modes of existence of the past in society, and that the way we use the past is essentially ideological and patrimonial. But the fact is that the past appears in a large number of works of art and practices devoid of any historicity, as an aesthetic and dramatic category. Working from empirical material (films, screenplays and role-playing games), we are able to observe how an interest in the past (with emphasis on the Middle Ages) may be quite remote from an interest in what has really happened.
\end{abstract}

Kewords : past versus history, modes of historicity, film, live action role-playing game, fiction, Middle Ages

Mots clés : passé versus histoire, régimes d'historicité, cinéma, jeu de rôles grandeur nature, fiction, Moyen Âge

Il existe une association, la Society for Creative Anachronism, dont le but est de recréer l'époque médiévale en fonction de la vision qu'elle a de cette période de l'histoire - et non pas de la réalité ${ }^{1}$.

Pour l'homme, le temps qui s'accumule comme à l'arrière du présent, et que la tradition européenne a mis en ordre, n'est pas seulement du passé. Il y a une actualité, une présence du passé dans notre société. Les historiens contribuent à cette "présence» en produisant un discours scientifique; certains d'entre eux s'attachent désormais à comprendre comment les sociétés pensent, utilisent, expérimentent le passé et plus largement le temps. Quelles relations une culture établit-elle à un moment donné entre passé, présent et futur? Cette « sémantique des temps historiques » ou l'analyse

1. Chuck Palahniuk, Confessions in Stone, dans Stranger than Fiction : True Stories, New York, Doubleday, 2005. Cf. le site de la Society for Creative Anachronism (http://www.sca.org). 
des « régimes d'historicité », pour reprendre deux titres d'ouvrages de référence, le premier de Reinhart Koselleck $(1979)^{2}$, le second de François Hartog $(2003)^{3}$, a mis en évidence qu'avant le XIX ${ }^{\mathrm{e}}$ siècle l'intelligibilité du présent provenait du passé, que l'expérience moderne était plutôt tournée vers l'avenir, et que depuis quelques décennies l'histoire semble s'actualiser dans le présent perpétuel du patrimoine et du souvenir ${ }^{4}$. Les historiens sont plus généralement préoccupés par les usages idéologiques et publics du passé dans une perspective critique de conformité à la réalité historique supposée ${ }^{5}$. Mais dans toutes ces réflexions, dominent les notions de représentation plutôt que d'expérience, et d'articulation plutôt que de présence, de réception et de création, qui président au contraire aux études sur le médiévalisme ${ }^{6}$.

\section{Pourquoi le passé?}

Aussi se propose-t-on de mettre l'accent sur ce qui a tendance à échapper à l'approche historisante des expériences du temps : le fait que le passé est une catégorie esthétique et dramatique à part entière. Pour ce faire, on va considérer deux domaines où la référence médiévale et plus largement la référence au passé est frappante et où il n'est pourtant pas tellement question d'histoire. Il s'agit du cinéma et du jeu, deux activités culturelles majeures de notre temps. En effet, que ce soit au cinéma, chez les scénaristes, dans la culture ludique et même dans le cas des reconstitutions historiques, l'intérêt ou la passion pour l'histoire tient une

2. Reinhart Koselleck, Le Futur passé. Contribution à la sémantique des temps historiques [1979], Paris, Éditions de l'EHESS, 1990.

3. François Hartog, Régimes d'historicité. Présentisme et expériences du temps, Paris, Seuil, 2003, en particulier p. 11-30. La notion de régimes d'historicité est suggérée pour la première fois par Hartog dans " Marshall Salhins et l'anthropologie de l'histoire», Annales. ESC, $n^{\circ}$ 6, 1983, p. 1256-1263. Voir aussi id. « Régimes d'historicité », Vox Poetica (www.voxpoetica/entretiens/hartog.html).

4. Cf. Pierre Nora (dir.), Les Lieux de mémoire, Paris, Gallimard, 1997, 3 vol.; Paul Ricœur, La Mémoire, l'histoire, l'oubli, Paris, Seuil, 2000.

5. Par exemple: Jànos Bak, Jörg Jarnut, Pierre Monnet et Bernd Schneidmüller (dir.), Gebrauch und Missbrauch des Mittelalters, 19.-21. Jahrhundert / Usages et Mésusages du Moyen Âge du XIX $x^{e}$ au XXI siècle, Munich, Fink, 2009 (titre cependant réducteur par rapport aux contributions); Christian Delacroix, François Dosse et Patrick Garcia (dir.), Historicités, Paris, La Découverte, 2009; Claire Andrieu, Marie-Claire Lavabre et Danielle Tartakowsky (dir.), Politiques du passé : usages politiques du passé dans la France contemporaine, Aixen-Provence, Presses universitaires de Provence, 2006; François Hartog et Jacques Revel (dir.), Les Usages politiques du passé, Paris, Éditions de l'EHESS, 2001.

6. Cf. Nathalie Koble et Mireille Séguy, Introduction à Passé présent. Le Moyen Âge dans ses fictions contemporaines, Paris, Éditions Rue d'Ulm, 2009, p. 7-23; Vincent Ferré, Introduction au présent volume : "Médiévalisme et théorie : pourquoi maintenant? »; la bibliographie internationale recensée par l'association Modernités médiévales (www.modernitesmedievales. org/bibliographie.htm). 
place assez minoritaire par rapport au passé purement visuel, d'ambiance, ou par rapport aux possibilités de récits et de mondes fictionnels qu'offre tout contexte non contemporain. Dans quantité d'œuvres et de pratiques, le passé est présent sans historicité.

Il s'agit de montrer la nécessité d'une séparation conceptuelle entre histoire et passé dans notre rapport au temps, de même qu'on a distingué histoire et mémoire, comme deux modes radicalement différents de rapport au passé. L'histoire, si l'on reprend la définition de Jacques Le Goff, relève de l'objectivité voire de la justice; la mémoire, elle, relève du mythe et de l'appropriation ${ }^{7}$. Un troisième champ devrait être établi, celui du passé sans l'histoire. Les usages non historiques du passé, voilà ce que l'on entend éclairer ici à partir d'un matériau empirique.

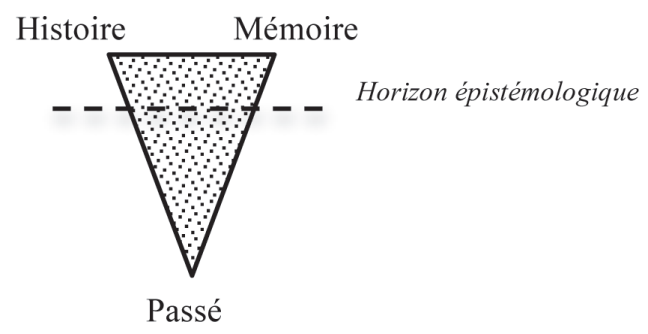

On ne prend pas ce point de départ en raison d'une certaine familiarité avec le raisonnement historique mais parce que ce dernier détient une position supérieure dans l'ordre épistémologique, dans le système de valeur académique ${ }^{8}$. Également parce que cette domination symbolique est parfois ressentie par les spécialistes des formes contemporaines (artistiques et populaires) du passé dans la culture. Enfin parce que le tabou de l'historien est double. D'une part, accepter qu'il éprouve un plaisir esthétique, «bien peu scientifique» au contact des œuvres du passé 9 . D'autre part, reconnaître à ce dernier des possibilités pour la vie actuelle, un site d'inspiration intellectuelle et pratique ${ }^{10}$.

7. Jacques Le Goff, Histoire et Mémoire, Paris, Gallimard, 1998, p. 194 et passim.

8. "Notre génération a la possibilité d'accueillir sans honte [...] ce "faux Moyen Âge", comme objet moderne digne d'étude » (Séverine Abiker et Florence Plet-Nicolas, préface à Le Moyen Âge en jeu, dirigé par les mêmes et Anne Besson, Eidôlon, ${ }^{\circ}$ 86, 2009, p. 10).

9. Ibid. Un sentiment fréquemment évoqué, également par les non-historiens. Par exemple Thomas Pavel, La Pensée du roman, Paris, Gallimard, 2003, p. 11.

10. Complexe analysé par Jeff Rider dans le présent volume; proposition développée par Siegfried Kracauer : «Le moi qui, revenu du passé, reprend le contrôle, se trouve enrichi des observations qu'il a pu faire pendant son retrait momentané » (L'Histoire des avant-dernières choses [1969], Paris, Stock, 2006, p. 154). C'est tout l'intérêt de l'ouvrage de Bruce Holsinger, The Premodern Condition. Medievalism and the Making of Theory, Chicago/ Londres, The University of Chicago Press, 2005, autour de Bataille, Lacan, Bourdieu, Derrida et Barthes. 


\section{L'historiocentrisme}

Le cinéma est un cas excellent parce que le témoignage des praticiens démontre à quel point l'intérêt pour le passé peut être étranger à l'intérêt pour l'histoire, et parce qu'en même temps, le point de vue « historien »y est institutionnalisé.

Pour ne retenir que deux exemples de l'approche historienne, Robert Rosenstone, un des pionniers des études du rapport entre cinéma et histoire aux États-Unis, s'inscrit dans le courant des études consacrées à la construction filmique de l'histoire ${ }^{11}$. En 1998, dans un essai «sur la possibilité réelle de transcrire l'Histoire en film », il conteste à l'écrit d'être la seule façon légitime de décrire le passé, avant de donner en 1995, dans «Like writing history with lighting. Film historique / vérité historique $^{12}$ », les règles pour réaliser un film «vraiment» historique, pour mettre en scène le passé. Rosenstone explique comment les cinéastes peuvent s'y prendre pour être des historiens, bien qu'on se situe dans le cadre de la fiction. Marc Ferro est sans doute le cas le plus important, le plus influent en France et en Allemagne, de la lecture historienne du couple image et histoire. Contribuant à faire de l'image, en particulier filmique et fictionnelle, un véritable document pour l'historien et voyant en elle un agent de l'histoire ${ }^{13}$, Ferro établit en 1987 « une classification globale des films dans leur rapport à l'histoire ${ }^{14} »$. Sous la forme d'un tableau à double entrée, il distingue quatre types de « discours sur la société » qu'une œuvre culturelle peut véhiculer : celui de l'idéologie institutionnalisée ou du discours officiel, celui de l'opposition qui peut proposer une contrehistoire, celui de la mémoire sociale et historique, celui de l'interprétation indépendante et originale; et quatre modes d'approche : par le haut, c'està-dire en observant les instances du pouvoir, par le bas si la question sociohistorique est évoquée par les masses, du dedans si l'auteur est investi dans son analyse et fait preuve d'autoréflexivité, enfin du dehors quand le contexte idéologique est reconstitué.

En réaction à cette avancée qui disqualifiait la critique positiviste sur les «bonnes» et les «mauvaises» représentions de l'histoire, s'est développée une approche proprement filmique, substituant l'analyse des

11. Robert A. Rosenstone, Visions of the Past : the Challenge of Film to our Idea of History, Cambridge, Mass., Harvard University Press, 1995; Id. (ed.), Revisioning History. Film and the Construction of a New Past, Princeton, N.J., Princeton University Press, 1995.

12. Id., "History in Images/History in Words : Reflections on the Possibility of Really Putting History onto Film », The American Historical Review, n 93/5, 1988, p. 1173-1185; Vingtième siècle, $\mathrm{n}^{\circ}$ 46/1, 1995, p. 164-166.

13. Cf. Marc Ferro, « Le film, une contre-analyse de la société? », Annales. ESC, n 1, 1973, p. 109-124: « partir de l'image, des images. Ne pas chercher seulement en elles illustration, confirmation, ou démenti...» (p. 113).

14. Id., «Y a-t-il une vision filmique de l'Histoire », Hors Cadre, ${ }^{\circ}$ 5, 1987, repris dans Cinéma et Histoire, nouvelle édition refondue, Paris, Gallimard, 1993, p. 217-266. 
formes «politiques » de l'histoire au cinéma par l'analyse des formes cinématographiques du passé. Passant du paradigme de la vision de l'histoire au cinéma à celle de l'écriture de l'histoire par le cinéma ${ }^{15}$, la question du passé dans la création cinématographique n'en est pas moins restée historiocentrée puisqu'il s'agit à présent de « déterminer la vertu historique » des films ${ }^{16}$, de mettre en avant les « capacités historiennes » du cinéma. L'histoire-caméra d'Antoine de Baecque (2008) ${ }^{17}$ est symptomatique à cet égard : or on voit parfaitement dans cet ouvrage la confusion notionnelle, du moins la non-distinction énonciative, entre histoire et passé. Le premier terme est souvent pris pour l'autre ${ }^{18}$, alors que - de Baecque le sait par ailleurs fort bien - l'histoire est toujours le récit du passé, le résultat d'une conscience de ce qui s'est passé, et que le passé est cette réalité disparue dont l'existence est toujours déjà médiatisée.

L'approche historique du passé est si prégnante ${ }^{19}$ qu'on peine à imaginer que les cinéastes puissent avoir pour le passé un intérêt autre que l'histoire quand ils ont recours à des situations non actuelles. Pourtant, si on se place du point de vue des praticiens, on est frappé par tout ce qu'il manque à la taxinomie « globale » du rapport cinématographique au passé. Face au passé, le cinéma est bien autre chose aussi qu'un moyen de propagande ou un mode d'écriture de l'histoire. Le passé filmique n'est pas seulement historien, politique, mémoriel ou mythologique. Il est même, comme on va le voir, essentiellement autre chose : un dispositif de création, un champ d'inspiration et d'expression à la fois formelles et idéelles à part entière. Si le passé ou plutôt les passés sont sollicités, c'est en grande partie pour leur force expressive, leurs qualités esthétiques, leur intérêt dramatique.

\section{La raison dramatique}

Toute une culture dramatique en effet dépend du passé. Depuis les plus anciens récits qui nous sont parvenus, à commencer par les mythes des origines, l'épopée de Gilgamesh au $\mathrm{III}^{\mathrm{e}}$ millénaire ou L'Iliade, l'art

15. Dans laquelle s'inscrit Rosenstone, History on Film/Film on History, New York, Pearson Longman, 2006.

16. Christian Delage et Vincent Guigueno, L'Historien et le Film, Paris, Gallimard, 2004, p. 24.

17. Antoine de Baecque, L'Histoire-caméra, Paris, Gallimard, 2008.

18. Ibid., « faire récit de l'histoire » (p. 12); « les trois modes de représentation de l'histoire » (p. 13); « les formes cinématographiques de l'histoire » (p. 19, titre de l'introduction), «l'armée de Spartacus, figurante de l'histoire» (p. 29), etc.

19. «Qu'est-ce qui est "perdu et gagné" quand le cinéma représente le passé? Comment réalisateurs et historiens s'accordent-ils sur la question de la vérité? L'émotion de l'événement historique au cinéma sert-elle à la compréhension du passé? ", quatrième de couverture de Richard Francaviglia et Jerry Rodnitzky (eds.), Lights, Camera, History. Portraying the Past in Film, College Station, Texas A\&M University Press, 2007. 
de raconter est intimement lié à la profondeur historique, sans que celle-ci soit l'objet ou la raison du récit. Le passé fournit à l'inspiration créatrice un grand nombre d'histoires, toutes plus ou moins déterminées par le moment où elles ont été produites. Certaines histoires ne sont tout simplement racontables que dans un cadre historique particulier, exactement comme l'isolement rend possible le récit d'horreur. Ainsi, pas de Source (1960) pour Ingmar Bergman et Ulla Isaksson sans la vaste campagne suédoise du $\mathrm{XIV}^{\mathrm{e}}$ siècle et sans le conte médiéval dont ils s'inspirent. Pas de Partition inachevée pour piano mécanique (1977) pour Nikita Mikhalkov sans la crise de la société russe de la fin du XIX ${ }^{\mathrm{e}}$ siècle et sa mise en comédie par Tchekhov dans les mêmes années. Le passé présente également des traits « mentaux » qui offrent des situations dramatiques particulières.

On peut ainsi inverser la question : non plus se demander quelle histoire donnent à voir les fictions, mais combien d'histoires ont été possibles grâce au passé, grâce par exemple aux sociétés européennes et méditerranéennes fondées sur l'honneur, sur la réputation, sur la vengeance. Bref, combien de récits de cinéma et de littérature les sociétés anciennes ont-elles rendues possibles?

Dans les écoles de cinéma et les ateliers d'écriture de scénario universitaires ou amateurs, le passé (éloigné de plusieurs siècles ou immédiat) représente une part non négligeable des projets ${ }^{20}$. Autour de $30 \%$. Cette proportion comprend des diégèses non actuelles (la Seconde Guerre mondiale, les années 1930, Vienne en 1914, Juin 1880, la Conquista en 1536, Sarajevo en 1995, l'Italie de 1482, la première croisade, etc.) et des univers-thèmes repris à l'imaginaire d'une période antérieure (le Graal, Avalon, Tristan et Iseut, le diable médiéval, Faust), auquel s'ajoute $8 \%$ d'univers alternatifs de genre (la science fiction, mais surtout la Fantasy). Trente pour cent des scénarios qui présentent des mondes inactuels s'inspirent d'œuvres non contemporaines (Chrétien de Troyes, Marcel Pagnol, Edgar Alan Poe, Stephan Zweig, les Weird Tales), conservant presque toujours l'époque de l'œuvre. Les déclarations d'intention montrent que le choix du passé, à quelques rares exceptions près, n'est jamais conditionné par un désir de connaissance ou de reconstitution de type historien. Et il arrive que l'historicité soit ressentie comme une contrainte et discutée par rapport à la liberté créatrice. Dans les motivations du recours au passé, une grande place est faite au visuel, à l'ambiance (le style Jazz ou l'univers policier), ainsi qu'au potentiel dramatique de

20. On se fonde ici sur les témoignages et les travaux de fin d'études de l'Institut supérieur des arts du spectacle (INSAS), de l'Institut des arts de diffusion (IAD) à Bruxelles et, tout spécialement, de la formation en écriture et analyse cinématographiques à l'Université Libre de Bruxelles (entre 1990 et 2008). Pour cette dernière institution, je remercie François Frédéric et Laurence Pousseur d'avoir rendu possible la consultation intensive des quelque deux cent cinquante scénarios et notes d'intention conservés dans les réserves de la bibliothèque des sciences humaines de l'ULB. 
tel événement ou de tel système de valeur, le poids des conventions par exemple; mais aussi, au facteur biographique et affectif : l'immigration des parents, l'épisode colonial de la famille, l'identité individuelle prise dans la tragédie collective. Il s'agit plus rarement de parler du monde actuel par un détour euphémique, profitant d'une situation passée pour traiter d'une question contemporaine, comme Bergman le fit en 1957 avec Le Septième Sceau pour évoquer la menace atomique. En somme, dans la grande majorité des scénarios, le cadre historique n'est ni l'objet d'un argument, ni l'objet du film. "C'est une tentation pour les cinéastes, dit Éric Rohmer, même ceux qui ne s'intéressent pas tellement à l'histoire, de faire des films historiques ${ }^{21}$. »

La critique considère généralement le « film d'histoire » comme un discours, et c'est là une partie du problème : les œuvres qui semblent avoir un lien avec le passé, ne serait-ce que par l'apparence, sont perçues d'emblée comme un discours sur l'histoire. Cela revient à attribuer à la création une intentionnalité qui lui est souvent étrangère. En effet, l'utilisation du passé par les cinéastes et les scénaristes est parfois strictement " esthétique », avec une volonté affichée de distance, de neutralité, de se servir du caractère exogène des situations pour exprimer une vérité humaine qui transcende précisément l'histoire.

Le cinéma de Terrence Malick - Days of Heaven (1978), The Thin Red Line (1998) qui met en scène la guerre du Pacifique, ou The New World (2006) qui se déroule à la fin du XVI ${ }^{\mathrm{e}}$ siècle - est caractéristique de ce complexe intentionnel, au sein même d'un cinéma hyper-reconstitutionniste et archéologisant, comme a pu l'être le cinéma d'Andreï Tarkovski. L'œuvre de Malick opère, au cœur même de la grande histoire, le dépassement des contingences historiques. Le cinéaste développe un propos humaniste auquel on peut adhérer ou non, une théologie d'inspiration spinoziste, où tous les êtres (hommes, bêtes et plantes) sont des déterminations provisoires d'une même âme. La même position ressort d'Apocalypse Now (1979). Relatant des faits appartenant à un passé très déterminé (la guerre du Vietnam qui s'achève en 1975), Francis Ford Coppola repose la question essentialiste que Joseph Conrad posait déjà, pour une époque également déterminée, dans Au cour des Ténèbres (1899) : l'homme a-t-il en partage la civilisation ou la barbarie?

\section{Surtout ne pas reconstituer : réalisme versus réalité}

Le cinéphile comme le savant interprètent généralement la composante historique d'un film sur le seul mode de l'historicité (au niveau le plus simple, par la recherche de l'« erreur» historique, de l'anachronisme).

21. Éric Rohmer à Priska Morrisey, Historiens et Cinéastes : rencontre de deux écritures, Paris, L'Harmattan, 2004. 
Il y a là quelque chose d'étrange. Cet a priori selon lequel toute réalité passée ne devrait être qu'historique et ne plus servir qu'à l'histoire et au souvenir.

Pourtant si l'on aborde de front la question de la reconstitution, on constate que les auteurs veulent avant tout créer une réalité et non pas « reconstituer » ou « reconstruire » le passé proprement dit; et chacun d'eux le fait selon des stratégies très différentes. Celles-ci ne nous apprennent rien sur le passé ou sur la manière d'écrire l'histoire, elles nous apprennent au contraire quelque chose sur notre culture de la réalité : qu'est-ce qui nous fait penser qu'une chose est vraie ou authentique? comment une réalité illusoire peut-elle faire illusion de réalité? C'est la question principale de ceux qui font des « films en costumes », pour prendre l'ancienne formule. À travers le " cinéma d'histoire ", parce que le cinéaste doit composer tout entier des mondes vraisemblables et sensibles, nous voyons de quelle manière se pense ce qui fait la réalité d'un monde. Le questionnement original à développer à partir des films qui situent leur récit dans un contexte non actuel, est alors celui des conceptions sensibles ou des représentations de la réalité, tel que Erich Auerbach et Thomas Pavel l'ont entrepris chacun à sa façon à partir du roman mondial ${ }^{22}$.

En d'autres termes, une interrogation sur la manière dont les univers historiques sont investis par les cinéastes en tant que moyen de recherche cinématographique est fondamentale si l'on veut comprendre le passé au cinéma (et cela vaut pour certains romans dits historiques). Pour que ressorte nettement la dimension non historienne des créations filmiques liées au passé, voici juxtaposées les réflexions de quelques cinéastes qui ont travaillé de manière très diverse, tout au long du siècle.

Carl Dreyer, à propos de La Passion de Jeanne d'Arc (1928) ou de Dies Irae (1943), explique en 1950 que « puisque le réalisme n'est pas l'art en soi, et puisqu'il faut en même temps qu'il y ait une correspondance entre l'authenticité des sentiments et l'authenticité des choses, [il] cherche à faire entrer les réalités dans une forme simplifiée et abrégée pour atteindre ce qu' [il] appellerai[t] un réalisme psychologique ${ }^{23} »$ :

[...] d'avance j'ai été conscient des exigences propres à ce projet $[\mathrm{La}$ Passion]. Traiter le sujet à la manière des films à costumes aurait peut-être pu permettre de décrire le $\mathrm{XV}^{\mathrm{e}}$ siècle et son environnement culturel, mais cela n'aurait abouti qu'à susciter une comparaison avec d'autres époques. Il s'agissait au contraire de faire en sorte que le spectateur fût absorbé par le passé ${ }^{24}$.

22. Erich Auerbach, Mimesis. La représentation de la réalité dans la littérature occidentale [1946], Paris, Gallimard, 1977; Thomas Pavel, op. cit.

23. Carl Th. Dreyer à Karl Roos (23 oct. 1950), « Ma seule grande passion », Réflexions sur mon métier, Paris, Cahiers du cinéma, 1983, p. 77.

24. Id., « La mystique réalisée », ibid., p. 41. 
En 1967, Pier Paolo Passolini crée avec Edipo Re une expérience de l'altérité historique. Loin de tout souci de fidélité, il rend une époque, comme on rend un son, par le syncrétisme et l'amalgame d'esthétiques culturelles. La culture matérielle de Medea (1969) est faite de la rencontre des costumes traditionnels d'Afrique, du Moyen-Orient et d'Amérique du Sud. Le monde mythique est également créé par la réinvention de sa géographie : Colchide et Corinthe sont respatialisées entre l'Italie (Trieste et Pise) et la Syrie (Alep), la Turquie (en Cappadoce). Il revendique cette dimension fictionnelle dans un entretien :

Je n'ai rien voulu reconstruire du point de vue archéologique et philologique. Je n'ai lu [...] aucun texte critique ou historique [...] concernant ce «moyen âge » grec où je voulais situer l'histoire. J'ai tout inventé ${ }^{25}$.

Roberto Rossellini, notamment dans Francesco, giullare di Dio (1952), qui met en scène la vie de saint François d'Assise, cherchait à révéler la nature humaine :

Une plus grande curiosité pour les individus. [...] Un besoin sincère, aussi, de voir avec humilité les hommes tels qu'ils sont, sans recourir au stratagème d'inventer l'extraordinaire. [...] Un désir, enfin, de s'éclairer soi-même et de ne pas ignorer la réalité... Pour nous comptait la recherche de la vérité, la correspondance avec le réel ${ }^{26}$.

« De cette façon [le néo-réalisme], on peut s'approcher de tout... Que le film soit en costume ou non n'a aucune importance. » L'homme est «petit, perdu, idiot, naïf. Et il fait de grandes choses». Selon Rossellini nous correspondons si bien à cette « double mesure » inscrite dans « le sentiment héroïque de la vie ${ }^{27}$ », qu'à l'éloignement du passé se substitue une étrange proximité. Non pas que l'histoire se répète, mais que c'est toujours le même homme qui souffre, endure, s'élève. L'histoire montre des constantes à travers la vie humaine - c'est là le rapport de Rossellini au passé, semblable à celui d'un certain nombre d'écrivains que l'approche historienne a tendance à qualifier de poétique de la répétition, du « retour du même » alors qu'il s'agit de réitération ${ }^{28}$. Des constantes existentielles produisent du présent dans le passé et des présences du passé dans le présent.

25. Pier Paolo Pasolini à J.-A. Fieschi, «Entretien avec Pasolini », Cahiers du cinéma, n 195,1967 , p. 13.

26. Roberto Rossellini à Mario Verdone, « Colloquio sul neo-realismo », Bianco e nero, février 1952, cité par Mario Verdone, Roberto Rossellini, choix de textes et propos de Rossellini, Paris, Seghers, 1963, p. 25-26.

27. Id. à Claude-Jean Philippe (oct. 1966), Le Cinéma révélé. Roberto Rossellini [1984], textes réunis par Alain Bergala, Paris, Flammarion, 2008, p. 130 et 131.

28. C'est le cas de Jean-François Hamel à propos de l'œuvre de Claude Simon : Revenances de l'histoire. Répétition, narrativité, modernité, Paris, Minuit, 2006, p. 178 sq. 
Enfin, Andreï Tarkovski, pour Andreï Roublev (1969), œuvre unanimement jugée comme un sommet de la reconstitution historique au même titre que Barry Lindon (1975) de Stanley Kubrick, voulait « récréer l'univers » de la Russie du $\mathrm{XV}^{\mathrm{e}}$ siècle "pour des yeux du $\mathrm{Xx}^{\mathrm{e}}$ siècle». Comme il l'écrit dans Le Temps scellé:

[...] il s'agissait d'imaginer un décor où le spectateur ne ressente d'aucune manière quelque impression de monument ou de musée: ni dans les costumes, ni dans l'expression orale, ni dans les mœurs, ni dans l'architecture. [...] La convention fut néanmoins inévitable, mais en tout cas à l'opposé de celle de la peinture animée. Si un spectateur du Xve siècle avait surgi, sans doute aurait-il trouvé la scène bien étrange [...]. C'est que nous vivons dans notre siècle et que nous n'avons plus le matériau d'il y a six cents ans. [...] telle fut d'ailleurs notre approche de la réalité [...] pour atteindre à cette vérité de l'observation directe, on pourrait dire à sa vérité psychologique, il nous a même fallu nous éloigner parfois de la vérité archéologique ou ethnographique ${ }^{29}$.

Dans ces quelques exemples, temps et espaces sont rapprochés : autres temps, autres lieux, le passé est comparé à un ailleurs. Ce rapprochement suggère que le problème qui se pose aux cinéastes n'est pas un problème de réalisme mais de "réalité ». Si certains sont plus doués que d'autres, c'est qu'ils montrent mieux ou traduisent plus efficacement ce qui constitue pour eux « la réalité » derrière le passage du temps, et non pas parce qu'ils sont les meilleurs historiens au cinéma. La réalisation est à prendre ici au pied de la lettre ${ }^{30}$.

\section{Le passé esthétique}

Il faut bien reconnaître qu'il est réducteur d'interroger notre culture ou sensibilité historique sans séparer les notions de passé et d'histoire. Qui se munit des questions traditionnelles de l'historien - les œuvres reproduisent-elles fidèlement l'époque? quelle vision de l'histoire véhiculent-elles? - risque en effet de passer à côté de ce qui est probablement le principal mode d'existence du passé dans les œuvres contemporaines. C'est aussi ce que montrent les jeux de rôles ${ }^{31}$, en particulier le jeu de rôles grandeur nature ${ }^{32}$.

29. Andreï Tarkovski, Le Temps scellé, Paris, Éditions de l'Étoile/Cahiers du cinéma, 1989, p. 72-73. 30. On trouvera des développements complémentaires dans Gil Bartholeyns, « Representation of the Past in Films : Between Historicity and Authenticity », Diogenes, ${ }^{\circ} 189,2000$, p. 31-47; «Représentation du passé au cinéma. Entre historicité et authenticité », Diogène, n 189 , 2000, p. 41-61.

31. Pour le jeu vidéo massivement multi-joueurs, voir Alexis Léonard, «Le Moyen Âge de loin : création et créativité autour des jeux vidéo ", dans Séverine Abiker et al., op. cit. (http://lapril.u-bordeaux3.fr/spip.php?article323).

32. On s'appuie ici sur des enquêtes menées depuis 2006, entretiens et sondages (quantitatifs 
Le jeu de rôles grandeur nature, inventé à la fin des années 1970 pour vivre « en vrai » les aventures qu' on vivait mentalement au cours d'un jeu de rôles sur table, se développe à partir des années 1990 et compte aujourd'hui plusieurs centaines d'associations à travers la plupart des pays occidentaux. Elles proposent divers styles de jeu, de l'intrigue politique en alcôve à la bataille rangée en plein champ, le plus souvent dans des univers dits " médiévaux-fantastiques », pendant quelques heures ou plusieurs jours, en petit groupe ou à plusieurs milliers. Cette activité, qu'il est préférable de comparer aux jeux à rôles auxquels on se livre dans l'enfance, plutôt qu'à du théâtre improvisé sans public, est parfois confondue avec la reconstitution historique par les médias et par les personnes qui croisent des rôlistes au cours d'une promenade. La confusion de ce jeu avec le phénomène des reconstitutions (lié à la valorisation patrimoniale des régions) est due à plusieurs éléments : la dimension costumée, les lieux investis (ruine de château, vieux moulin), la proximité des deux communautés de pratiquants, et un développement européen relativement synchrone ${ }^{33}$.

Malgré ces liens avec cette histoire au présent, les créations "grandeur nature » ne reposent sur aucune composante mémorielle ou commémorative. On y rencontre le passé par l'atmosphère et par la culture matérielle : c'est un passé aspectuel. De façon comparable avec le cinéma ${ }^{34}$, le lien entre passé et jeu de rôles est de plusieurs ordres, mais il n'est pas en priorité historisant ou passéiste. Sur plusieurs centaines de praticiens (auteurs, créateurs, animateurs et joueurs confondus), seulement $11 \%$ disent pratiquer cette activité pour la « passion de l'histoire » ou pour vivre comme au Moyen Âge, et ceux-ci sont généralement des adolescents ou des étudiants en histoire ${ }^{35}$. Une étude similaire sur l'activité de

et qualitatifs) réalisés aux printemps 2007 et 2009. Cf. Gil Bartholeyns et Daniel Bonvoisin, « Le Moyen Âge sinon rien. Statut et usage du passé dans le jeu de rôle grandeur nature », dans Élodie Burle-Errecade et Valérie Naudet (dir.), Fantasmagories du Moyen Âge. Entre médiéval et moyenâgeux, Senefiance, $\mathrm{n}^{\circ}$ 54, 2010, p. 47-57, version longue : www.modernitesmedievales.org/articles/XBonvoisinJdRGN.htm, 2007; Gil Bartholeyns, « Manières de faire des mondes grandeur nature », dans Séverine Abiker et al., op. cit., p. 30-42, version longue : http://lapril.u-bordeaux3.fr/spip.php ?article415. Gil Bartholeyns et Daniel Bonvoisin, « Les créations ludiques contemporaines sont-elles solubles dans le patrimoine? Culture et communatué du jeu de rôles grandeur nature », dans Chiara Bortolotto et Sylvie Grenet (dir.), Le Patrimoine culturel immatériel, Paris, Éditions de la Maison des sciences de l'homme, 2010. $I d$., « Portrait du GN : communauté, valeur et culture. Les résultats d'une deuxième enquête Internet », dans Larp.eu. An International and Open Community dedicated to Live-action Roleplaying Theory, www.larp.eu, à paraître. Voir aussi infra : « Le Gn : par qui... ».

33. Gil Bartholeyns et Daniel Bonvoisin, "Les créations ludiques contemporaines... », op. cit.

34. Cf. dans ce volume l'article de Corneliu Dragomirescu qui reflète notre projet en cours d'élaboration : A Feeling of the Past. The Cinema Before the Age of Cinema.

35. L'ensemble des résultats sous forme de graphiques est paru dans Gil Bartholeyns et Daniel Bonvoisin, «Le GN : par qui, comment, pourquoi. Les résultats d'une enquête Internet », 
reconstitution historique montrerait sans doute que l'intérêt pour l'histoire représente lui aussi une part seulement de la motivation des praticiens. Comme pour le jeu de rôles grandeur nature, la convivialité, la dimension festive et l'évasion, la créativité matérielle et le développement personnel tiennent une place conséquente dans cette pratique de l'histoire - pour parler en toute rigueur, on devrait dire : dans cette pratique $d u$ passé.

Le Moyen Âge est un phénomène esthétique et un cadre pragmatique en raison de références largement partagées, généralement déjà médiatisées par des œuvres de fictions qui relèvent souvent de la fantasy ${ }^{36}$, et en raison de la facilité de réalisation. Un rien - une clairière, une tour, une cape - et nous sommes comme par défaut au Moyen Âge, sans que cette période historique soit l'environnement où l'on est projeté, dans lequel on est immergé. Si les pratiquants devaient plonger quelqu'un en deux minutes dans un univers médiéval, comment s'y prendraient-ils? «Des bougies, des draps aux murs, chemise médiévale ou tabard pour tout le monde », ou « Dans la forêt, avec une lampe-tempête, une cape et des poursuivants à ses trousses ${ }^{37} \gg$.

Cette présence du passé en l'absence de l'histoire, ce que beaucoup appellent le « Médiéval », est plus qu'un genre. Ce substantif indique à lui seul qu'il ne s'agit pas d'une actualisation historique, même fantasmée, mais d'un opérateur ludique, et avant cela même, d'une fonction créatrice d'univers et de récits : le Médiéval comme représentation civilisationnelle liée à la guerre, à la sorcellerie, au banquet... pour ne prendre que quelques « sémaphores » qui reviennent fréquemment quand les pratiquants expliquent à quoi renvoie pour eux, ludiquement, le Moyen Âge. En somme, le rapport d'usage entre univers ludiques et passé est un rapport fondé sur la collection de références susceptibles d'être partagées et matérialisées. Le fait que ce principe vaille pour toutes les créations d'univers (futuristes ou les « ailleurs » absolus) montre que l'historicité du passé n'est nullement impliquée dans la production et la viabilité des univers qui semblent relever du passé. C'est une question de culture de mondes plus que de culture historique. Selon les personnes, l'immensité de l'histoire humaine offre plus ou moins de situations qui font ou qui peuvent faire monde ${ }^{38}$. Le passé est d'abord un pourvoyeur d'univers non historiques.

S'il est bien établi que, pour le jeu, comme pour le cinéma, passé et histoire gagnent à être distingués afin de rendre compte de la nature du passé dans les œuvres culturelles de notre époque (mais sans doute aussi dès

Larp.eu. An International and Open Community dedicated to Live-action Roleplaying Theory, (www.larp.eu).

36. La dimension « merveilleuse » et « magique » des univers moyenâgeux est parfois spontanément justifiée par le fait qu'au Moyen Âge, on croyait à la sorcellerie et aux monstres... 37. Respectivement : chimiste de 26 ans; enseignant de 33 ans; étudiant de 30 ans : « Quelques tentures, de l'obscurité [...] ou simplement costumées au milieu d'un bois » (enquête de 2007). 38. Cf. Gil Bartholeyns, « Manières de faire des mondes grandeur nature », op. cit. 
l'apparition du médiévalisme artistique et social et spécialement dans celui du XIX ${ }^{e}$ siècle), on peut se demander ce qui fait la « supériorité » manifeste du Moyen Âge sur les autres périodes, à quoi répond le Moyen Âge aujourd'hui dans la société occidentale. Avant d'y voir une articulation essentielle de la modernité, avant d'y voir la catégorie historique de l'altérité ${ }^{39}$ - au même titre que l'Orient ou l'Afrique incarnent la catégorie de l'altérité culturelle, et l'animal celle de l'altérité anthropologique - il y a la dimension esthétique au sens large. Dans le jeu, du moins, c'est avant tout la facilité d'exécution collective et la liberté pour l'imagination qui sont invoquées : parce que "c'est dans notre culture ", "parce que cet univers est associé à tous les possibles », ou parce qu'il est mal connu et qu'on peut donc y mettre tout ce que l'on veut. La sensibilité des joueurs à l'égard du Moyen Âge est radicalement orientée « récit», «drame», «potentiel d'aventures », en dépit du niveau de connaissance historique, parfois élevé, qu'ils manifestent. Monde de légendes. Monde sonore où l'on festoie. Monde de périls et de troubles. Monde héroïque et secret ${ }^{40}$. De sorte que les pratiquants parlent du Moyen Âge comme s'ils répondaient à cette question : qu'est-ce que le passé permet dès lors qu'il n'est pas historique?

\section{Histoire/mémoire, passé/histoire}

Comme synthèse on propose la situation schématique suivante, qui rend compte de façon plus équilibrée du rapport que la société entretient avec le temps.

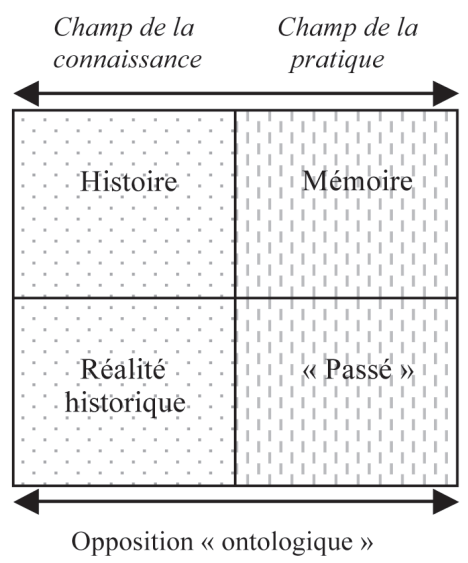

Au point de vue conceptuel, l'histoire est définie contre la mémoire. La mémoire est séparée de l'histoire par le procès épistémologique propre à

39. Sur ces deux aspects, voir en particulier Paul Zumthor, Parler du Moyen Âge, Paris, Minuit, 1980.

40. Cf. Gil Bartholeyns et Daniel Bonvoisin, « Le Moyen Âge sinon rien », op. cit. 
la démarche historique. L'histoire est le lien de connaissance avec la réalité historique, une réalité que l'historien vise à établir ou à rétablir, contre l'histoire officielle, nationale, ethnocentrique, contre l'histoire mythique, l'horizon d'attente propre à l'histoire-mémoire.

La mémoire et le passé (au sens développé ici de «passé sans histoire ») ont en commun qu'ils sont essentiellement des pratiques sociales et culturelles ${ }^{41}$. Le passé, défini comme pratique non historique, et la mémoire partagent le statut d'état historique dégradé aux yeux de l'historien idéal-type. Cet historien comprend leurs manifestations comme des représentations, selon la vieille tradition platonicienne de l'image comme reflet, comme manque d'être. Ainsi, il dira que le cinéma véhicule des « images » plus ou moins fidèles du passé et, comme Siegfreid Kracauer l'a montré ${ }^{42}$, il refusera l'idée selon laquelle le cinéma produit lui aussi un discours historique, est lui-même un mode de connaissance du passé.

Le passé existe largement dans un rapport d'extériorité à l'histoire. Il se distingue du couple histoire-mémoire dans la mesure où il est dénué ou faiblement chargé d'idéologie (intentionnelle) et d'intérêt pour ce qui s'est passé. Les statues ou les fêtes commémoratives relèvent du mythe national, les travaux universitaires relèvent de l'historiographie. Mais les romans d'un Italo Calvino, les jeux de rôles qui perdent leur raison d'être s'ils rejouent l'histoire, l'esthétique iconographique d'un Éric Rohmer, ou les réactivations littéraires de l'imaginaire médiéval sont inclassables en termes d'usage politique ou de vérité historique. Tous relèvent d'une troisième forme vivante du passé.

Gil Bartholeyns

Université Charles de Gaulle Lille 3

41. L'histoire l'est aussi, en plus d'être une pratique discursive, mais c'est un autre sujet. Cf. Michel De Certeau, L'Écriture de l'histoire, Paris, Gallimard, 1975, « L'opération historiographique », p. 77 sq.; Michel Foucault, Les Mots et les Choses, Paris, Gallimard, 1966, p. 378-385; Paul Ricœur, Temps et récit, I. L’intrigue et le récit historique, Paris, Seuil, 1983 ; Krzysztof Pomian, Sur l'histoire, Paris, Gallimard, 1999.

42. Siegfried Kracauer, Theory of Film. The Redemption of Physical Reality, Londres/Oxford/ New York, Oxford University Press, 1960; L'Histoire des avant-dernières choses [1969], op. cit. 